\title{
Eficacia de la doramectina vía intramuscular sobre nematodos gastrointestinales en ovinos (Ovis aries)
}

\author{
Efficacy of intramuscular doramectin on gastrointestinal nematodes \\ in sheep (Ovis aries)
}

\author{
Buitrago M, Jhonny ${ }^{\star 1}$ MVZ, Cardona A, José2 Ph.D, Montes V, Donicer ${ }^{3}$ Ph.D. \\ ${ }_{1}^{1}$ Universidad de Córdoba, Facultad de Medicina Veterinaria y Zootecnia, Programa de Maestría en Ciencias Veterinarias del \\ Trópico, Grupo de Investigación en Medicina de Grandes Animales (MEGA), Montería, Colombia. \\ ${ }^{2}$ Universidad de Córdoba, Facultad de Medicina Veterinaria y Zootecnia, Profesor Titular de Medicina y Clínica de Grandes \\ Animales, Grupo de Investigación en Medicina de Grandes Animales (MEGA), Montería, Colombia. \\ ${ }^{3}$ Universidad de Sucre. Facultad de Ciencias Agropecuarias, Departamento de Zootecnia. Sincelejo, Colombia.
}

\section{Key words:}

Ruminants; avermectins; gastrointestinal parasitism; treatment.

\section{Abstract}

Small ruminants are susceptible to helminth infections, and remain a major constraint on its activity; the main tool for controlling these pathogens is based on the administration of anthelmintic, but there is increasing development of resistance to these drugs, causing difficulty in control. This study evaluated the effectiveness of doramectin administered intramuscularly in sheep, 11 naturally infected sheep was taken and divided into two groups, a control group (CG) without treatment was constituted by 4 animals, and treated group (GT) were constituted for seven animals receiving doramectin $1 \%$. Both groups were sampled by Mc Master technique before treatment (TO) and 6,10,16,24 and 30 days post treatment. Descriptive statistics was performed and efficiency of treatment at different sampling times was calculated. $100 \%$ animals were parasitized at T0, and a low percentage of efficacy of treatment was taken during study days and a percentage of overall efficiency of $46.76 \%$ product, this indicates resistance doramectin

\section{Palabras Clave:}

Rumiantes; avermectinas; parasitismo gastroentérico; tratamiento.
INFORMACIÓN

Recibido: 10-12-2016; Aceptado: 21-03-2017. *Correspondencia autor: Jhabuitragome@hotmail.com

\section{Resumen}

Los pequeños rumiantes son susceptibles a las infecciones helmínticas, siendo una limitante importante en su actividad, la principal herramienta para el control de estos patógenos se basa en la administración de antihelmínticos, pero existe un creciente desarrollo de resistencias a estos fármacos, generando dificultad en su control. Este estudio evaluó la efectividad de la Doramectina administrada vía intramuscular en ovinos, para lo cual se emplearon 11 ovinos naturalmente infectados distribuidos en dos grupos, un grupo control (GC) sin tratamiento que fue constituido por 4 animales, y un grupo tratado (GT) conformado por 7 animales que recibieron doramectina al $1 \%$. Ambos grupos fueron muestreados mediante la técnica de Mc Master antes del tratamiento (T0) y a los días 6, 10, 16, 24 y 30 post tratamiento. Se realizó estadística descriptiva y se calculó la eficiencia del tratamiento a los distintos tiempos de muestreo. El 100\% de los animales se encontraban parasitados en el T0, y se tuvo un bajo porcentaje de eficacia del tratamiento durante los días del estudio y un porcentaje de eficacia general del producto del $46,76 \%$, esto indica la presencia de resistencia a la doramectina. 


\section{INTRODUCCIÓN}

En Colombia la cadena ovino caprina es relativamente joven, se conforma en su mayoría por pequeños productores y se encuentra dividida principalmente en dos sistemas de producción, el primero dedicado a la producción de cárnicos y productos artesanales y el segundo a la producción de leche y sus derivados, aunque una pequeña parte están dirigidos a proporcionar animales para pie de cría y lana (MINISTERIO DE AGRICULTURA Y DESARROLLO RURALOBSERVATORIO AGROCADENAS COLOMBIA, 2006; AREVALO, 2011)(), Según el Instituto Colombiano Agropecuario (ICA) (2016) la población ovina y caprina en Colombia se calcula en 1'423.274 animales distribuidos principalmente en los departamentos de La Guajira $(46,69 \%)$, Boyacá $(8,04 \%)$, Magdalena $(7,71 \%)$, Córdoba $(5,55 \%)$ y Cesar $(5,41 \%)$, mostrando que la cadena cuenta con una participación importante en la región de la Costa Atlántica, constituida por los departamentos de Guajira, Magdalena, Atlántico, Bolívar, Sucre y Córdoba, (MINISTERIO DE AGRICULTURA Y DESARROLLO RURALOBSERVATORIO AGROCADENAS COLOMBIA, 2006).

Desde el punto de vista sanitario los pequeños rumiantes revisten gran importancia al ser susceptibles a enfermedades epidemiológicamente importantes y ser decisivas en la supervivencia y diseminación de agentes virales, bacterianos o parasitarios. (ICA, 2016), en especial de las infecciones helmínticas que son consideradas las más prevalentes, patogénicas y económicamente más importantes de todas las enfermedades parasíticas de los pequeños rumiantes, (TRAVERSA \& VON SAMSON-HIMMELSTJERNA, 2016) con un riesgo mayor en sistemas de producción basados en pastoreo (LOPEZ et al., 2013).

En los sistemas de producción ovina las gastroenteritis parasitarias son consideradas como una limitante importante (QUIJADA, et al., 2006; ENSUNCHO et al., 2014) debido a la disminución en la eficiencia productiva de los animales afectados(PULIDO et al., 2014), siendo un problema de importancia médica y económica que debe ser objeto de atención especialmente en lo que concierne a su control (ENSUNCHO et al., 2014), en este sentido el conocimiento de la distribución y diversidad de la fauna parasitaria en los sistemas productivos es necesaria para establecer programas que minimicen este problema, así como las pérdidas económicas que ocasiona (PULIDO et al., 2014)

En países como México y Venezuela se han reportado altas prevalencias de parásitos gastrointestinales (QUIJADA et al., 2006; LOPEZ et al., 2013). En Colombia estudios realizados en Antioquia muestran una frecuencia de $86.6 \%$ (HERRERA et al., 2013) y una prevalencia de76\%, (ZAPATA et al., 2016), mientras que en Córdoba se observó un $97,7 \%$ de prevalencia(ENSUNCHO et al., 2014) y en Boyacá, en el municipio de Toca se encontró una frecuencia del 100\%(PULIDO et al., 2014)

Entre los helmintos que pueden afectar a los ovinos, los más relevantes son los estróngilos gastrointestinales por ser los de mayor impacto negativo (QUIJADA et al., 2006; TRAVERSA\& VON SAMSON-HIMMELSTJERNA, 2016) tanto a nivel sanitario como económico en países industrializados y en desarrollo (TRAVERSA \& VON SAMSON-HIMMELSTJERNA, 2016), las especies que se han reportado con mayor prevalencia a nivel mundial son Haemonchus contortus (ARCE \& RODRIGUEZ, 2010; HERRERA et al., 2013; LOPEZ et al., 2013; DIAZ et al., 2014; ENSUNCHO et al., 2014; ZAPATA et al., 2016). Trichostrongylus colubriformis (QUIJADA et al., 2006; ARCE \& RODRIGUEZ, 2010; LOPEZ et al., 2013). Oesophagostomum columbianum (ARCE \& RODRIGUEZ, 2010; HERRERA et al., 2013; ENSUNCHO et al., 2014) Teladorsagia (Ostertagia) circumcincta (HERRERA et al., 2013; ENSUNCHO et al., 2014; ZAPATA et al., 2016). Strongyloides spp. (ENSUNCHO et al., 2014) y Cooperia curticei (LOPEZ et al., 2013), encontrando asociación entre la especie causante de la infección y variables como sexo, sistema de producción, estado fisiológico, periodo de ocupación de potreros y rotación de potreros (ZAPATA et al., 2016).

En la actualidad la principal herramienta para el control de estos patógenos es la administración de antihelmínticos, aunque el desarrollo creciente de la resistencia a estos fármacos está comprometiendo la sustentabilidad de esta alternativa (ANZIANI \& MUCHIUT, 2014), por lo que se han reportado cambios en la emergencia y dificultad de control de una gran cantidad de infecciones parasíticas en ovinos en la última década.(TYLOR, 2012), por este motivo y debido a las altas prevalencias reportadas para el departamento de Córdoba, el presente estudio tuvo como objetivo evaluar la eficacia de la doramectina sobre los nematodos gastrointestinales en ovinos de esta región, y determinar su potencial uso como alternativa en el control del parasitismo gastroentérico Ovino.

\section{MATERIALES Y MÉTODOS}

El estudio fue de tipo experimental y se realizó en la granja de producción de la Facultad de Medicina Veterinaria y Zootecnia de la Universidad de Córdoba, ubicada en el municipio de Ciénaga de Oro, Córdoba, Colombia, a $7^{\circ} 23^{\prime}$ y $9^{\circ} 26^{\prime}$ de $L N$ y a los $74^{\circ} 52^{\prime}$ y $76^{\circ}$ $32^{\prime}$ de LO del meridiano de Greenwich, a una altura de $30 \mathrm{msnm}$, con temperatura promedio anual de $28^{\circ} \mathrm{C}$, 
humedad relativa del $82 \%$, precipitación media anual de $1400 \mathrm{~mm}$, perteneciente a la formación climática de bosque tropical lluvioso. Se presentan dos estaciones bien definidas (época de lluvia y época seca) (PABON et al., 2001).

Para la investigación se emplearon 11 ovinos criollos de levante, de ambos sexos, todos naturalmente infectados con parásitos gastrointestinales. Previo al estudio ninguno de los animales había recibido ningún tipo de tratamiento antiparasitario.

Se tomaron muestras de heces directamente de la ampolla rectal para determinar la carga parasitaria pretratamiento mediante la técnica de Mc Master (KLEI, 1986). Posteriormente los animales fueron distribuidos al azar en dos grupos. El grupo 1 o grupo control (GC) estuvo constituido por 4 animales que no recibieron tratamiento antihelmíntico, El grupo 2 o grupo tratado (GT) fue conformado por 7 animales que recibieron tratamiento con doramectina al 1\% (Edo-doramectina, Laboratorios Edo, Colombia) a dosis de $0,2 \mathrm{mg} / \mathrm{kg}$, dosis única, aplicada intramuscular profunda en el músculo glúteo medio.

Se realizó remuestreo de la materia fecal en los días 6 , $10,16,24$ y 30 post tratamiento para realizar recuentos de huevos por gramo (HPG) mediante la técnica de Mc Master

Los animales fueron mantenidos en semiestabulación, durante el día se les permitía pastoreo rotacional, mientras que al atardecer y en la noche se manejaban dentro del aprisco sumistrando forraje para ser consumido en estos periodos. El tratamiento fue aplicado en horas de la mañana sin previo ayuno. Las muestras coprológicas fueron transportadas refrigeradas al laboratorio de Parasitología de la Facultad de Medicina Veterinaria y Zootecnia de la Universidad de Córdoba, con el fin de realizar la Técnica cuantitativa de Mc Master (KLEI, 1986).

Se realizó una base de datos con los resultados obtenidos en ambos grupos a los distintos tiempos de muestreo y se realizó análisis estadístico descriptivo, determinando el número total de HPG de heces pos tratamiento. El porcentaje de eficacia se calculó mediante la fórmula, descrita por Powers et al. (POWERS, 1982), donde

Se usó la prueba de $t$-student, con el objeto de verificar si existían diferencias significativas entre los dos grupos (GC y GT) y los tiempos de muestreo $(P \leq 0.05)$.

\section{RESULTADOS Y DISCUSIÓN}

El control parasitario en la mayoría de las granjas se basa casi exclusivamente en el uso de antihelmínticos pertenecientes a distintos grupos farmacológicos como lo son las lactonas macrocíclicas, los bencimidazoles, las tetrohidropirimidinas-imidazotiazoles, los derivados de amino acetonitrilo y los espiroindoles, (TRAVERSA \& VON SAMSON-HIMMELSTJERNA, 2016).Sin embargo, este uso intensivo de antihelmínticos como medida terapéutica y de control de las gastroenteritis parasitarias ha causado la diseminación global de poblaciones parasitas resistentes (GONZALES et al., 2012; TRAVERSA \& VON SAMSONHIMMELSTJERNA, 2016;) TRAVERSA \& VON SAMSON-HIMMELSTJERNA, 2016),

Muchos nematodos de importancia en la Medicina Veterinaria poseen características genéticas que favorecen el desarrollo de resistencia antihelmíntica, lo que se ha vuelto uno de los asuntos de mayor interés en la producción con pequeños rumiantes (PAPADOPOULOS, 2008). Los parásitos que afectan a la especie ovina en los que más se reportado el fenómeno de resistencia antihelmíntica incluyen Haemonchus contortus, Teladorsagia circumcincta. Trichostrongylus spp.,(HOLSBACK et al., 2016; TRAVERSA \& VON SAMSON-HIMMELSTJERNA, 2016), Cooperia y Nematodirus, aunque este último es el menos relacionado con este fenómeno (HOLSBACK et al., 2016)

En las tabla I y II se observa que el $100 \%$ de los animales seleccionados para el estudio se encontraban parasitados, y que tanto el grupo control como el grupo tratado presentaban en promedio infecciones severas (mayores a 600 HPG) (ZAPATA et al., 2016) al día 0 . Adicionalmente se observa que en ambos grupos se presentó un nivel moderado a alto en todos los muestreos (valores mayores a $200 \mathrm{HPG}$ ) (ZAPATA et al., 2016), es necesario aclarar que en el grupo control se presentó un mayor conteo de huevos que en el grupo tratado y la cantidad de huevos encontrados tuvo una tendencia creciente durante el experimento. Llama la atención que en ambos grupos se encuentra

Tabla 1. Evolución en el conteo de HPG en el grupo de animales tratados con doramectina $1 \%$ por vía intramuscular a dosis de $0,2 \mathrm{mg} / \mathrm{kg}$.

\begin{tabular}{ccccccc}
\hline \multicolumn{7}{c}{ Tratados } \\
\hline Animal n $^{\circ}$ & día 0 & día 6 & día 10 & día 16 & día 24 & día 30 \\
\hline 1 & 140 & 100 & 50 & 40 & 20 & 0 \\
3 & 400 & 60 & 260 & 500 & 850 & 1480 \\
4 & 100 & 0 & 140 & 1310 & 1400 & 1620 \\
5 & 11600 & 12980 & 666 & 2720 & 120 & 0 \\
6 & 790 & 70 & 290 & 240 & 400 & 560 \\
7 & 810 & 60 & 880 & 3900 & 1160 & 3010 \\
Promedio & 2307 & 2212 & 381 & 1452 & 658 & 1112 \\
Desviación estándar & 4563 & 5275 & 323 & 1549 & 566 & 1164 \\
\hline \multicolumn{7}{c}{ Media geométrica general } \\
\hline
\end{tabular}


Tabla 2. Evolución de los HPG en el grupo de animales control.

\begin{tabular}{ccccccc}
\hline \multicolumn{7}{c}{ Control } \\
\hline Animal n $^{\circ}$ & día 0 & día 6 & día 10 & día 16 & día 24 & día 30 \\
\hline 1 & 3810 & 3580 & 1470 & 7400 & 12200 & 8770 \\
2 & 100 & 340 & 140 & 1310 & 1480 & 1620 \\
3 & 360 & 580 & 1090 & 2450 & 3540 & 3740 \\
4 & 670 & 890 & 800 & 1560 & 2050 & 2100 \\
Promedio & 1235 & 1347,5 & 875 & 3180 & 4817,5 & 4057,5 \\
Desviación estándar & 1732 & 1505 & 562 & 2856 & 4998 & 3270 \\
\hline \multicolumn{7}{c}{ Media geométrica general } \\
\hline \multicolumn{7}{c}{}
\end{tabular}

un aumento en la cantidad de huevos al día seis y una reducción hacia el día 10 para incrementarse nuevamente al día 16.

En la Figura 1 se observa que existe una tendencia general a la disminución de la cantidad de huevos por gramo en el grupo tratado, aunque nunca se alcanzaron niveles bajos en el conteo (inferiores a 200 HPG) (ZAPATA et al., 2016), mientras que en la Figura 2 se muestra que la tendencia en el grupo control es al aumento, manteniendo siempre niveles de infección superiores a los 1000 HPG.

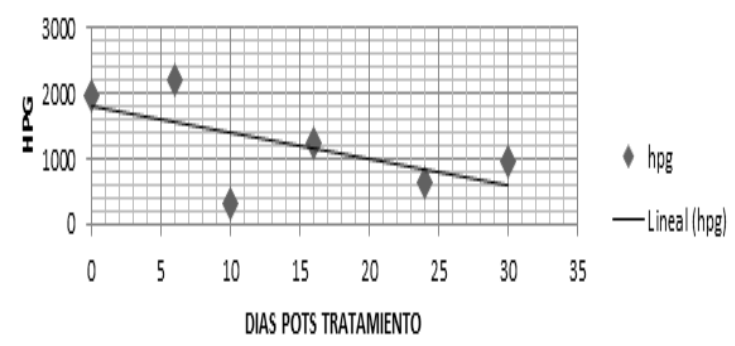

Figura 1. Tendencias de la cantidad de HPG en el grupo tratado con doramectina $1 \%$ a dosis de $0.2 \mathrm{mg} / \mathrm{kg}$.

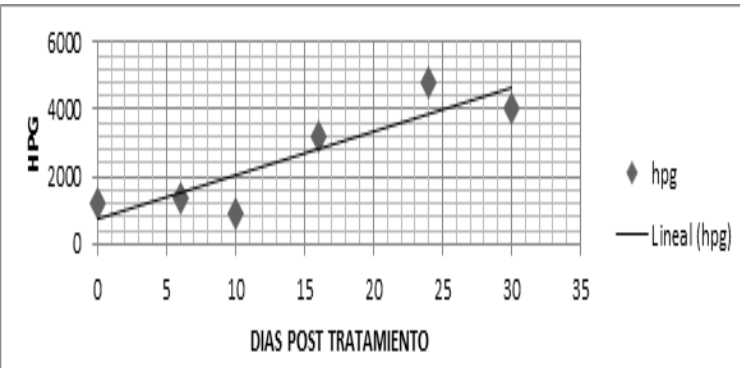

Figura 2. Tendencias de la cantidad de HPG en el grupo control.

En la tabla III, se observan los porcentajes de eficacia del tratamiento durante los días del estudio, en el cual se obtuvo un porcentaje de eficacia general del producto del $46,76 \%$. Se observa que existe una aparente mejoría en la eficacia al día 24 y 30 post tratamiento $(86,34 \%$ y $72,59 \%$ ), Lo anterior se se debe, a que se presentó un aumento del HPG en el grupo control en estos días y no por una disminución en el conteo de huevos del grupo tratado, lo que podría deberse a que exista una susceptibilidad diferenciada a la doramectina entre las especies parasitas disminuyendo así la ovoposición en este periodo en el grupo tratado o a un aumento de las poblaciones parasitas en el grupo control debido a una continua reinfección a partir del pasto, este aspecto requieren mayor investigación.

Tabla 3. Porcentaje de eficacia de la doramectina aplicada vía intramuscular en ovinos criollos en diferentes días pos tratamiento.

\begin{tabular}{cc}
\hline$\%$ eficacia & Días pos tratamiento \\
\hline$-64,15$ & 6 \\
56,45 & 10 \\
54,33 & 16 \\
86,34 & 24 \\
72,59 & 30 \\
\hline
\end{tabular}

La eficacia general se calculó de la siguiente manera:

$\%$ eficacia $=\frac{2118,9-1128}{2118,9} \times 100=46,76$

El resultado de este estudio muestra una baja eficacia de la doramectina para el control de los parásitos gastrointestinales en ovinos, y permite afirmar que existe resistencia a la doramectina en las especies parasitas que afectan de forma natural los ovinos en el departamento de Córdoba, pues Según las recomendaciones de la WAAVP (World Association for the Advancement of Veterinary Parasitology), existe resistencia al antihelmíntico administrado cuando el porcentaje de reducción del recuento de huevos es menor al $95 \%$. Estos resultados concuerdan con lo reportado por HOLSBACK et al. (2016) quienes encontraron una efectividad moderada de la doramectina contra Haemonchus spp, pero insuficiente contra Cooperia spp. Anteriormente SARRE et al. (2012) también habían reportado la resistencia a la doramectina de Haemonchs contortus y otras especies parasitas en alpacas.

En Colombia también se han reportado casos de resistencia a antihelmínticos (HERRERA et al., 2013), específicamente en el departamento de Antioquia en donde se encontró una alta proporción de animales positivos independiente del antihelmíntico utilizado, por lo que se reportó una protección parcial de los antihelmínticos independiente del principio activo.

Para la mayoría de grupos antihelmínticos también se ha reportado resistencia en grados variables 
(PAPADOPOULOS, 2008) encontrándose resistencias individuales a principios activos como la Ivermectina, Fenbendazol (TORO et al., 2014), Nitroxinil (HOLSBACK et al., 2016), Levamisol y Closantel (ANZIANI \& MUCHIUT, 2014), incluso se han descrito poblaciones de estróngilos capaces de sobrevivir a más de una clase de antihelmíntico (TRAVERSA \& VON SAMSON-HIMMELSTJERNA, 2016) así como a sus combinaciones (HOLSBACK et al., 2016). En Argentina, se reportaron especies de Haemonchus spp. con resistencia múltiple a Levamisol, Fenbendazol, Ivermectina y Closantel (ANZIANI \& MUCHIUT, 2014), en México, se reportó resistencia múltiple a Ivermectina y Levamisol (GONZALES et al., 2012), mientras que en Brasil, se encontraron nematodos con resistencia múltiple a Doramectina, Fenbendazol y Nitroxinil (Holsback, y otros, 2016), lo que pone en evidencia la ocurrencia y posible diseminación de las poblaciones de estróngilos resistentes a Bencimidazoles, Tetrahidropirimidinas, Imidiotazoles y con una menor extensión a las Lactonas macrocíclicas (TRAVERSA \& VON SAMSON-HIMMELSTJERNA, 2016). La excepción a la diseminación de este fenómeno estaría presente en Cuba, en donde se reporta una baja frecuencia de resistencias que ha sido atribuido al uso limitado de fármacos antihelmínticos en el país debido a razones económicas (MENCHO et al., 2013).

Como en este estudio no se realizó identificación de las especies parasitas que se encontraban infectando de forma natural a los ovinos, es necesario realizar evaluaciones posteriores que determinen si esta resistencia podría darse de manera diferencial entre las distintas especies parasitas, pues se ha encontrado asociaciones entre la especie identificada y la efectividad del principio activo utilizado, así como su frecuencia de uso (HERRERA et al., 2013; HOLSBACK et al., 2016). Es importante resaltar que durante el transcurso de este estudio no se observaron manifestaciones clínicas de intolerancia o de inflamación focalizada en ninguno de los animales tratados que pudiera sugerir algún proceso inflamatorio o reacción adversa al tratamiento con doramectina aplicada vía intramuscular.

La detección de estas resistencias es necesaria para poder diseñar estrategias de control que retrasen su desarrollo (PAPADOPOULOS, 2008) y aunque existen algunos métodos in vivo e in vitro, así como algunas herramientas moleculares disponibles para realizar esta detección (DEMELER et al., 2012), aún es necesario mejorarlos, particularmente en lo referente a la detección de resistencias a las lactonas macrocíclicas (COLES et al., 2006). También existe una clara necesidad de implementar programas de monitoreo para evaluar la eficacia de los antihelmínticos como requisito esencial para desarrollar programas de control parasitario efectivos (TORO et al., 2014). Siendo fundamental para ello el correcto diagnóstico de las especies parasíticas que estén afectando la producción (DEMELER et al., 2012), pues la emergencia de líneas de nematodos resistentes y la dependencia puesta en los antihelmínticos para su control pueden inducir grandes cambios en la epidemiologia de las infecciones parasitarias (TYLOR, 2012).

Los programas de control basados exclusivamente en el uso de antihelmínticos son anticuados (TRAVERSA\& VON SAMSON-HIMMELSTJERNA, 2016) y pueden constituir una amenaza para la producción ovina en muchas partes del mundo (TYLOR, 2012), por lo que es necesario programas de control con enfoques más sustentables que incluyan el uso integrado de medidas que garanticen la preservación de las moléculas que aún son efectivas y que minimicen la intensidad de la infección (TRAVERSA \& VON SAMSON-HIMMELSTJERNA, 2016), así como la presión de selección sobre los parásitos resistentes (ARCE \& RODRIGUEZ, 2010). Las tendencias actuales no solo tienen en cuenta los niveles de infestación parasitaria del rebaño, sino también la población larvaria en el pasto, denominada "población en refugio" (VAN WYK, 2001).

Otras opciones para el manejo del parasitismo incluyen el uso de razas resistentes, pero presentan como inconveniente que tienden a ser menos productivas, también se ha intentado con el uso de plantas que contengan taninos condensados $u$ otros compuestos activos, aunque esta opción no ha sido completamente evaluada. Hasta el momento ninguno de estos métodos parece ser suficiente sin el soporte antihelmíntico y por tanto no ofrecen una opción práctica, aunque es posible que reduzcan la dependencia en el uso de químicos (PAPADOPOULOS, 2008).

Independiente de la estrategia utilizada para el control parasitario es necesario capacitar a los productores acerca de la importancia de la correcta utilización de los antihelmínticos, así como las consecuencias de su uso indiscriminado (TORO et al., 2014), pues son ellos los directamente involucrados en tomar decisiones acerca del tipo y frecuencia de los tratamientos antiparasitarios (BEYNON, 2012).

\section{CONCLUSIONES}

Algunas de las especies parasitas que afectan a los ovinos en el Departamento de Córdoba ya están presentando resistencia a la Doramectina, es necesario realizar más investigación para establecer cuáles son las especies que presentan mayor resistencia y cuales aún se encuentran en estado susceptible y como sería la distribución de esta resistencia en la región para poder establecer programas de control parasitarios más efectivos para la especie. Es necesario además concientizar a los productores en nuevas estrategias orientadas al control parasitario que no se basen de manera exclusiva en el uso de antihelmínticos y que permitan la sostenibilidad a las producciones basadas en esta especie. 


\section{REFERENCIAS}

ANZIANI, O., \& MUCHIUT, S. (2014). antihelmíntica múltiple (closantel, febendazol, ivermectina y levamisol) en Haemonchus spp parasitando ovinos en la provincia de Santa Fe. Ineficacia de una triple combinación de estas drogas para su control. Revista de medicina veterinaria, 95(1), 00-00.

ARCE, J., \& RODRIGUEZ, J. G. (2010). Dinámica de las larvas infestantes de estrongílidos gastrointestinales en ovinos en pastoreo. Pastos y Forrajes, 33(1), 1-1.

AREVALO, S. (2011). LA CADENA CÁRNICA OVINA EN COLOMBIA Y URUGUAY. Tesis, Instituto Nacional de Investigación Agropecuaria del Uruguay - Universidad Nacional de Colombia, Tacuarembó-Uruguay.

BEYNON, S. A. (2012). Potential environmental consequences of administration of anthelmintics to sheep. Veterinary Parasitology, 189, 113-124.

COLES, G. C., JACKSON, F., POMROY, W. E., PRICHARD, R. K., VON SAMSON-HIMMELSTJERNA, G., SILVESTRE, A., Y OTROS. (2006). The detection of anthelmintic resistance in nematodes of veterinary importance. Veterinary Parasitology, 136, 167-185.

DEMELER, J., SCHEIN, E., \& VON SAMSON-HIMMELSTJERNA, G. (2012). Advances in laboratory diagnosis of parasitic infections of sheep. Veterinary Parasitology, 189, 52-64.

DIAZ ANAYA , A., ARIAS GONZALES , H., GARCIA CORREDOR, D., \& PULIDO MEDELLIN, M. (2014). Estimación de los Valores de Hematocrito y Hemoglobina en Presenciade Haemonchus sp. en Ovinos de Oicatá, Colombia. Rev. Fac. Cs. Vets., 55(1), 18-24.

ENSUNCHO HOYOS, C., CASTELLANO CORONADO, A., MAZA ANGULO, L., BUSTAMANTE YANEZ, M., \& VERGARA GARAY, O. (2014). prevalencia y grado de infección de nematodos gastrointestinales en ovinos de pelo en pastoreo de cuatro municipios de cordoba, colombia. Revista Científica, FCV-LUZ, 24(5), 414-420.

GONZALES GARDUÑO, R., TORRES HERNANDEZ, G., LOPEZ ARRELLANO, M., \& MENDOZA DE GIVES, P. (2012). Resistencia antihelmíntica de nematodos parásitos en ovinos. Revista de Geografía Agrícola(48-49), 62-72.

HERRERA O, L., RIOS, L., \& ZAPATA, R. (2013). Frecuencia de la infección por nemátodos gastrointestinales en ovinos y caprinos de cinco municipios de Antioquia. Rev.MVZ Córdoba, 18(3), 3851-3860.

HOLSBACK, L., RAMSEY LUPPI, P. A., SANCHES SILVA, C., KREMER NEGRAO, G., CONDE, G., VINICIUS GABRIEL, H. (2016). Anthelmintic efficiency of doramectin, fenbendazole, and nitroxynil, in combination or individually, in sheep worm control. Braz. J. Vet. Parasitol., Jaboticabal.

ICA, Instituto Colombiano Agropecuario. (2016). Censo Pecuario Nacional - 2016. Recuperado el 14 de jun de 2016, de ICA: http://www.ica.gov.co/getdoc/8232c0e5-be97-42bd-b07b-9cdbfb07fcac/Censos-2008.aspx

KLEI, T. (1986). Laboratory diagnosis. Vet Clin, N. Am. Equine Pract, 2, 381-393.

LOPEZ RUVALCABA, O. A., GONZALES GARDUÑO, R., OSORIO ARCE, M. M., ARANDA IBAÑEZ, E., \& DIAZ RIVERA, P. (2013). Cargas y especies prevalentes de nematodos gastrointestinales en ovinos de pelo destinados al abasto. Rev Mex Cienc Pecu, 4(2), 223-234.

MENCHO PONCE , J. D., GUERRA LLORENS, Y., PADILLA VILLAVICENCIO, L., RODRIGUE DIEGO, J. G., \& BELTRAO MOLENTO, M. (2013). Eficacia antihelmíntica de la Ivermectina 1\% (Labiomec $囚$ ) en rebaños ovinos de Camagüey, Cuba. Rev. Salud Anim., 35(2), 134-136.

MINISTERIO DE AGRICULTURA Y DESARROLLO RURAL-OBSERVATORIO AGROCADENAS COLOMBIA. (2006). DOCUMENTO DE TRABAJO No. 125: LA CADENA OVINOS Y CAPRINOS EN COLOMBIA . Ministerio de Agricultura y Desarrollo Rural, Bogotá, Colombia. 
PABON, J., ESLAVA, J., \& GOMEZ, R. (2001). Generalidades de la distribucion espacial y temporal de la temperatura del aire y de la precipitacion en Colombia. Meteorologia colombiana, 4, 47-59.

PAPADOPOULOS, E. (2008). Anthelmintic resistance in sheep nematodes. Small Ruminant Research, 76, 99-103.

PEREZ, R., PALMA, C., CABEZAS, I., RUBILAR, L., \& ARBOIX, M. (2010). The influence of gastrointestinal parasitism on fecal elimination of doramectin in lambs. Ecotoxicology andEnvironmentalSafety, 73, 2017-2021.

POWERS K, W. L. (1982). World associations for advancement of veterinary parasitology (W.A.A.V.P) Guidelines for evaluating the efficacy of anthelmintics in rumiants (bovine and ovine). Vet Parasitol, 10, 265-284.

PULIDO MEDELLIN, M. O., GARCIA CORREDOR, D., DIAZ ANAYA, A., \& ANDRADE BECERRA, R. (2014). Pesquisa de parásitos gastrointestinales en pequeñas explotaciones ovinas del municipio de Toca, Colombia. Rev. Salud Anim., 36(1), 65-69.

QUIJADA, J., GARCIA, F., VIVAS ISIS, SIMOES, D., \& RONDON, Z. (2006). PREVALENCIA DE INFECCIONES POR ESTRÓNGILOS DIGESTIVOS EN UN REBAÑO OVINO DEL ESTADO ARAGUA EN LA ÉPOCA DE LLUVIA. FCV-LUZ, 16(4), 341-346.

SARRE, C., CLAEREBOUT, E., VERCRUYSSE, J., LEVECKE, B., GELDHOF, P., PARDON, B. (2012). Doramectin resistance in Haemonchus contortus on an alpaca farm in Belgium. Veterinary Parasitology, 185, 346-351.

TORO, A., RUBILAR, L., PALMA, C., \& PEREZ, R. (2014). Resistencia antihelmíntica en nematodos gastrointestinales de ovinos tratados con ivermectina y fenbendazol. Arch Med Vet, 46, 247-252.

TRAVERSA, G., \& VON SAMSON-HIMMELSTJERNA, G. (2016). Anthelmintic resistance in sheep gastro-intestinal strongyles in Europe. Small Ruminant Research(135), 75-80.

TYLOR, M. A. (2012). Emerging parasitic diseases of sheep. Veterinary Parasitology, 189, 2-7.

VAN WYK, J.A. (2001). Refugia-overlooked as perhaps the most potent factor concerning the development of anthelmintic resistance. Onderstepoort Journal of Veterinary Research. 66(1), 55-.67.

ZAPATA SALAS, R., VELASQUEZ VELEZ, R., HERRERA OSPINA, L. V., RIOS OSORIO, L., \& POLANCO ECHEVERRY, D. N. (2016). Prevalencia de Nematodos Gastrointestinales en Sistemas de Producción Ovina y Caprina bajo Confinamiento, Semiconfinamiento y Pastoreo en Municipios de Antioquia, Colombia. Rev Inv Vet Perú, 27(2), 344-354. 